\title{
SELETIVIDADE DE AGROTÓXICOS UTILIZADOS EM POMARES DE PÊSSEGO A ADULTOS DO PREDADOR Chrysoperla externa (HAGEN, 1861) (NEUROPTERA: CHRYSOPIDAE) ${ }^{1}$
}

\author{
RODOLFO VARGAS CASTILHOS 2 , ANDERSON DIONEI GRÜTZMACHER ${ }^{3}$, \\ DORI EDSON NAVA ${ }^{4}$, MOISÉS JOÃO ZOTTI ${ }^{5}$, PAULO RICARDO BAIER SIQUEIRA ${ }^{6}$
}

RESUMO - A seletividade de dezesseis agrotóxicos utilizados na produção integrada e convencional de pêssego foi avaliada sobre a fase adulta de Chrysoperla externa através de bioensaios de exposição residual conduzidos em laboratório (temperatura de $25 \pm 1^{\circ} \mathrm{C}$, umidade relativa $70 \pm 10 \%$ e fotofase 14 horas), utilizandose de metodologia prescrita pela "International Organization for Biological Control" (IOBC). Os agrotóxicos (\% de ingrediente ativo na calda) azoxystrobina $(0,016)$, captana $(0,192)$, dodina $(0,126)$, folpete $(0,200)$, mancozebe $(0,256)$, mancozebe + oxicloreto de cobre $(0,140+0,096)$, tebuconazole $(0,320)$, abamectina $(0,002)$, óleo mineral $1(2,420)$, óleo mineral $2(1,920)$, dicloreto de paraquate $(0,300)$ e glifosato $(1,440)$ foram inócuos; deltametrina $(0,002)$ foi levemente nocivo e dimetoato $(0,160)$, fosmete $(0,160)$ e malationa $(0,240)$ foram nocivos a adultos de $C$. externa.

Termos para indexação: Controle biológico, crisopídeo, agrotóxicos, Prunus persica L.

\section{SELECTIVITY OF PESTICIDES USED IN PEACH ORCHARD ON ADULTS OF Chrysoperla externa (HAGEN, 1861) (NEUROPTERA: CHRYSOPIDAE)}

\begin{abstract}
The selectivity of sixteen pesticides used in integrated and conventional peach production was evaluated on adults of Chrysoperla externa (Hagen,1861) (Neuroptera: Chrysopidae) under bioassays in laboratory conditions (temperature of $25 \pm 1{ }^{\circ} \mathrm{C}$, relative humidity $70 \pm 10 \%$ and 14 hours photophase), using the "International Organization for Biological Control" (IOBC) methodology. The pesticides (\% active ingredient) azoxystrobin (0.016), captan (0.192), dodine (0.126), pholpet (0.200), mancozeb (0.256), mancozeb + cooper oxichloride $(0.140+0.096)$, tebuconazole $(0.320)$, abamectin $(0.002)$, mineral oil $1(2.420)$, mineral oil 2 (1.920), paraquat dichloride $(0.300)$ and gliphosate (1.440) were harmless; deltametrin $(0.002)$ was slightly harmful and dimetoate $(0.160)$, phosmet $(0.160)$ and malathion $(0.240)$, were harmful to $C$. externa adults. Index terms: Biological control, lacewing, chemicals, Prunus persica L.
\end{abstract}

\footnotetext{
${ }^{1}$ (Trabalho 060-10). Recebido em: 01-03-2010. Aceito para publicação em: 19-11-2010. Realizado com apoio financeiro do CNPq e da CAPES.

${ }^{2}$ Eng. Agr., Mestrando do Programa de Pós-Graduação em Fitossanidade (PPGFs)/FAEM/UFPel Câmpus Universitário Cx. P. 354 96010-900 Pelotas-RS. E-mail: rvcastilhos@hotmail.com;

${ }^{3}$ Eng. Agr., Dr., Prof. Depto. de Fitossanidade/FAEM/UFPel. Câmpus Universitário Cx. P. 354 - 96010-900 Pelotas-RS. E-mail: anderson.grutzmacher@pq.cnpq.br;

${ }^{4}$ Eng. Agr.,Pesquisador da Embrapa Clima Temperado. Rodovia BR 392, km 78 Cx. P. 403 - $96001-970$ Pelotas-RS. E-mail: nava@cpact.embrapa.br;

${ }^{5}$ Eng. Agr.,Doutorando do PPGFs/FAEM/UFPel. Câmpus Universitário Cx. P. 354 - 96010-900 Pelotas-RS E-mail: zottimoises@yahoo.co.uk ${ }^{6}$ Acadêmico de Graduação em Agronomia da FAEM/UFPel, Bolsista do CNPq. Câmpus Universitário Cx. P. 354 - $96010-900$ PelotasRS. E-mail: agrosiqueira@yahoo.com.br
} 


\section{INTRODUÇÃO}

A cultura do pessegueiro possui relevante importância para o Estado do Rio Grande do Sul, que produz anualmente 129.000 toneladas de pêssego, aproximadamente $54 \%$ da produção nacional (IBGE, 2010). Entretanto, a ocorrência de insetos-praga, principalmente mosca-das-frutas Anastrepha fraterculus (Wiedemann, 1830) (Diptera: Tephritidae) e da mariposa oriental Grapholita molesta (Busck, 1916) (Lepidoptera: Tortricidae), dentre outros fatores, limitam a produção de pêssegos (BOTTON et al., 2005), fazendo com que o RS apresente rendimentos médios inferiores quando comparados a outros estados.

Apesar dos avanços obtidos com o sistema de Produção Integrada de Pêssego (PIP), que prioriza a adoção de técnicas de controle preconizadas no Manejo Integrado de Pragas (MIP), como utilização de métodos naturais e realização de monitoramento (NTEPIP, 2009), a pulverização por calendário de inseticidas de amplo espectro de ação ainda se constitui na medida de controle mais adotada pelos persicultores. Esta acarreta, entre outros problemas, o surgimento de pragas secundárias, como pulgões, ácaros e cochonilhas, em função da eliminação dos inimigos naturais, causando desequilíbrio ecológico (BOTTON et al., 2005).

Uma vez que o uso de agrotóxicos ainda é o método de controle de pragas mais utilizado na cultura do pessegueiro, faz-se necessária a utilização de produtos que sejam seletivos aos insetos benéficos, entre os quais se incluem os inimigos naturais das pragas, para que assim o MIP na cultura seja viabilizado (CARVALHO, 2002). Para isso, é indispensável a realização de testes de seletividade, a fim de se classificarem os compostos quanto ao efeito sobre estes insetos e assim facilitar a tomada de decisão sobre qual produto utilizar.

O predador Chrysoperla externa (Hagen, 1861) (Neuroptera: Chrysopidae) é um inseto polífago e é comumente encontrado em diversos agroecossistemas (DE BORTOLI et al., 2006), inclusive em pomares de pessegueiro (SCHUBER et al., 2008), constituindo-se em um importante inimigo natural de ácaros, cochonilhas, pulgões e ovos de lepidópteros.

Trabalhos de seletividade com esta espécie foram realizados para frutíferas, como macieira (MOURA et al., 2009) e citros (GODOY et al., 2004), porém para a cultura do pessegueiro não foram encontrados até o momento trabalhos na literatura. A maioria dos estudos de seletividade com $C$. externa foram realizados para a fase larval do predador, que é mais suscetível aos agrotóxicos, sendo a fase adulta (geralmente menos suscetível) muitas vezes renegada em trabalhos desta natureza.

Neste contexto, estudos de seletividade sobre a fase adulta deste predador são de suma importância, uma vez que, para se mensurar o efeito de um agrotóxico sobre determinada espécie, faz-se necessário realizar testes sobre todas as diferentes fases do desenvolvimento, principalmente se for considerado que a fase adulta é responsável pela dispersão e oviposição nos pomares. Desta maneira, o trabalho teve como objetivo avaliar a seletividade de agrotóxicos utilizados na produção integrada e convencional de pêssego sobre adultos (machos e fêmeas) do predador C. externa, em bioensaios conduzidos em laboratório.

\section{MATERIAL E MÉTODOS}

Os bioensaios foram conduzidos no Laboratório do Núcleo de Manejo Integrado de Pragas (NUMIP), da Embrapa Clima Temperado, e nos laboratórios de Controle Biológico e de Pesticidas, da Universidade Federal de Pelotas, Capão do Leão-RS, a partir de uma adaptação da metodologia estabelecida pela "International Organization for Biological Control" (IOBC) para a espécie Chrysoperla carnea (Stephens, 1836) (Neuroptera: Chrysopidae) (VOGT, 2001).

Os insetos utilizados nos bioensaios foram provenientes de uma criação massal estabelecida em laboratório (temperatura de $25 \pm 1^{\circ} \mathrm{C}$, umidade relativa $70 \pm 10 \%$ e fotofase 14 horas), conforme descrito por Carvalho e Souza (2000) e Vogt et al. (2000).

Foram avaliados, sobre insetos adultos de C. externa, 16 agrotóxicos (Tabela 1) registrados no AGROFIT (2008) para a cultura do pessegueiro, sendo a grande maioria recomendos na PIP.

Cada bioensaio foi composto por quatro agrotóxicos, uma testemunha negativa (ausência de agrotóxico) e um tratamento-padrão de reconhecida toxicidade, tal como o inseticida fenitrotiona $(\mathrm{Su}-$ mithion 500 EC) (MOURA, 2007). As dosagens utilizadas (Tabela 1) foram as máximas recomendadas para a cultura nas normas da PIP, ajustadas para corresponder a um volume de calda de 800 L.ha $^{-1}$.

Os bioensaios consistiram na exposição de adultos de C. externa a resíduos secos dos agrotóxi$\cos$, que foram pulverizados sobre placas de vidro $(12 \times 12 \mathrm{~cm})$, utilizando-se de um pulverizador pressurizado a $\mathrm{CO}_{2}$, com bico de aplicação de jato plano uniforme (Teejet XR110015EVS). A pressão de trabalho utilizada na pulverização foi de aproximadamente 50 psi, o que correspondeu a um depósito de calda de $2 \pm 0,2 \mathrm{mg} . \mathrm{cm}^{-2}$, conforme metodologia preconizada pela IOBC/WPRS (VOGT, 2001). 
Após a secagem da calda, as placas de vidro pulverizadas foram levadas para o laboratório, onde serviram de fundo e cobertura na confecção das gaiolas para exposição dos insetos. Cada gaiola foi composta por um anel de metacrilato $(10 \mathrm{~cm}$ de diâmetro x $3 \mathrm{~cm}$ de altura), com 5 orifícios de $1,3 \mathrm{~cm}$ de diâmetro, fechados com tecido tipo "voile" para permitir a ventilação, um orifício com as mesmas dimensões para conexão de uma bomba de aquário modelo Big-air A 420 com fluxo invertido, trabalhando na velocidade média para sucção de vapores tóxicos e um orifício menor $(0,8 \mathrm{~cm})$, por onde foi fornecida água aos insetos. A dieta dos adultos, composta por $15 \mathrm{~mL}$ de leite condensado, 2 gemas de ovo, 1 clara de ovo, $30 \mathrm{~g}$ de mel, $20 \mathrm{~g}$ de açúcar, $30 \mathrm{~g}$ de levedura de cerveja, $50 \mathrm{~g}$ de germe de trigo e $45 \mathrm{~mL}$ de água destilada, foi fornecida lateralmente na gaiola, em quantidade suficiente para a realização do bioensaio.

Após a confecção das gaiolas, adultos do predador previamente separados por sexo, com aproximadamente uma semana de idade, foram adicionados às gaiolas de exposição. Cada tratamento consistiu em quatro gaiolas contendo cada uma cinco casais, sendo cada gaiola considerada uma repetição no delineamento inteiramente casualizado. A mortalidade acumulada de machos e fêmeas, assim como a mortalidade geral foram avaliadas às 24; 72 e 120 horas após a exposição dos insetos aos agrotóxicos.

Os valores obtidos referentes ao número de insetos mortos foram submetidos à análise de variância; e as médias, comparadas pelo teste de Tukey, a $5 \%$ de significância, pelo programa estatístico WinStat (MACHADO; CONCEIÇÃO, 2007). As porcentagens de mortalidade foram calculadas para cada tratamento e corrigidas em função da testemunha pela fórmula de Schneider-Orelli (PÜNTENER, 1981), sendo os produtos classificados em cada período de avaliação, de acordo com índices propostos pela IOBC em: 1) inócuo $(<30 \%)$; 2) levemente nocivo $(30-79 \%)$; 3) moderadamente nocivo (80-99\%); e 4) nocivo ( $>99 \%)$, em que a classificação final do composto foi aquela atribuída às 120 horas após a pulverização dos agrotóxicos.

\section{RESULTADOS E DISCUSSÃO}

Fêmeas e machos de C. externa apresentaram diferenças na mortalidade quando expostos a resíduos dos agrotóxicos. De modo geral, percebeu-se que a mortalidade se mostrou mais acentuada nos insetos machos. Este fato pôde ser observado para os inseticidas fosmete e dimetoato, onde se constatou, na primeira avaliação (24 horas após a exposição), significativa diferença entre o número de machos e fêmeas mortos, semelhante ao ocorrido para o padrão de toxicidade (fenitrotiona) nos bieonsaios I e II (Tabela 2).

A mortalidade de machos foi superior à mortalidade de fêmeas quando adultos foram expostos a resíduos do inseticida piretroide deltametrina no bioensaio II, em todas as avaliações, sendo a média de insetos mortos às 120 horas após a exposição de 2,8 e 0,5 para machos e fêmeas, respectivamente. Para o fungicida mancozebe + oxicloreto de cobre no bioensaio IV, a maior suscetibilidade de machos também foi detectada em todos os períodos de avaliação, sendo esta mais acentuada a partir de 72 horas após a exposição (Tabela 2). O mesmo ocorreu quando adultos foram expostos ao herbicida glifosato, que proporcionou discrepância na mortalidade de machos e fêmeas, que foi de 1,5 e 0,3 inseto, respectivamente, às 120 horas após a exposição (Tabela 2).

Uma vez que a taxa de exposição de machos e fêmeas aos agrotóxicos foi a mesma, a maior sobrevivência das fêmeas nos tratamentos deve-se ao dimorfismo sexual encontrado em adultos. Além das diferenças sexuais primárias, adultos de C. externa apresentam diferenças nas dimensões corporais, sendo as fêmeas de maior tamanho em relação aos machos (CARVALHO; SOUZA, 2000). Esta diferença de tamanho explica, em parte, o fato de as fêmeas serem mais resistentes, e assim apresentarem menor mortalidade ou levarem mais tempo para sucumbirem ao efeito dos agrotóxicos quando comparadas com os machos. Castro et al. (2009) registraram uma longevidade de 46,57 e 71,50 dias para machos e fêmeas de C. externa, respectivamente, demonstrando que, além da menor suscetibilidade aos agrotóxicos, as fêmeas possuem naturalmente maior longevidade em relação aos machos.

De todos os trabalhos de seletividade encontrados na literatura com esta espécie, nenhum mostrou a diferença entre a mortalidade ocorrida em machos e fêmeas de $C$. externa, sendo este detalhe renegado em estudos desta natureza. Porém, a diferenciação entre a mortalidade de machos e fêmeas em estudos deste tipo é imprescindível, pois pode resultar em importantes informações para o manejo integrado de insetos-praga, uma vez que a menor suscetibilidade das fêmeas a certos agrotóxicos pode contribuir para a manutenção da população deste inimigo natural em um agroecossistema.

Com relação às mortalidades e à classificação de toxicidade dos agrotóxicos às 24; 72 e 120 horas após a exposição residual dos adultos nos quatro bioensaios (Tabela 3), pôde-se observar que os inseticidas organofosforados fosmete e dimetoato ocasionaram uma mortalidade inicial (24 horas após exposição) de $80 \%$, proporcionando mortalidade total a partir de 72 horas após o início da exposição, sendo enquadrados na classe 4 (nocivo). Do mesmo 
modo, malationa, pertencente ao mesmo grupo químico, apresentou-se nocivo a adultos do predador, porém apresentando $100 \%$ de mortalidade somente 120 horas após o início da exposição. A mortalidade ocasionada por estes compostos foi semelhante à mortalidade apresentada pelo padrão de toxicidade fenitrotiona nos quatro bioensaios (Tabela 3 ).

Resultados obtidos por Giolo et al. (2009), com a espécie $C$. carnea em testes de exposição residual, assemelham-se aos obtidos no presente estudo para o inseticida dimetoato. Os autores obtiveram $100 \%$ de mortalidade para a espécie, comprovando a alta toxicidade deste composto. Entretanto, fosmete foi classificado como moderadamente nocivo (classe 3), com uma mortalidade de $91,4 \%$, o que provavelmente se deve à menor concentração de ingrediente ativo utilizada no referido estudo e também a diferenças de suscetibilidade entre as duas espécies. A toxicidade de inseticidas pertencentes ao grupo químico dos organofosforados foi confirmada também por Silva et al. (2006), que obtiveram 100\% de mortalidade em adultos de $C$. externa pulverizados com clorpirifós.

Os resultados obtidos com o inseticida/ acaricida abamectina, que não causou mortalidade sobre $C$. externa (Tabela 3), são semelhantes aos obtidos por Moura (2007), que classificou o referido ingrediente ativo como inócuo a duas populações de $C$. externa e por Godoy et al. (2004), que verificaram $100 \%$ de sobrevivência em adultos do predador pulverizados com este composto. Giolo et al. (2009) também não obtiveram mortalidade para a espécie $C$. carnea quando esta foi exposta a resíduos de abamectina. Já Vilela (2009) obteve $63 \%$ de mortalidade em adultos pulverizados diretamente com o produto em concentração de ingrediente ativo equivalente à utilizada no presente estudo, classificando-o como levemente nocivo aos adultos de $C$. externa.

Quanto à classificação de toxicidade, o inseticida piretroide deltametrina apresentou-se inócuo (classe 1) às 24 e 72 horas após o início da exposição, com uma mortalidade de $2,5 \%$ e 7,5\%, respectivamente; porém, às 120 horas, o mesmo ocasionou mortalidade de $32,5 \%$ sendo classificado como levemente nocivo (classe 2 ) a adultos de $C$. externa (Tabela 3). De forma similar, Giolo et al. (2009) verificaram uma mortalidade de 3,1\% em C. carnea 72 horas após a exposição a resíduos de deltametrina. Resultados distintos foram obtidos por Godoy et al. (2004), que constataram 100\% de mortalidade quando adultos de $C$. externa receberam pulverização direta de deltametrina e por Silva et al. (2006), que obtiveram $82,5 \%$ de mortalidade para o inseticida betaciflutrina, pertencente ao mesmo grupo químico.
As duas formulações à base de óleo mineral mostraram-se inócuas (classe 1) a adultos do predador em todas as avaliações, com mortalidade variando de 0,0 a 5,0\% (Tabela 3). Valor distinto foi encontrado por Rocha (2008), que verificou mortalidade de 14,3\% em adultos que receberam óleo mineral através de pulverização direta. As diferentes metodologias utilizadas nos estudos podem explicar as discrepâncias nas taxas de mortalidade proporcionadas para uma espécie por um mesmo ingrediente ativo, o que evidencia a importância da utilização de metodologias padronizadas da IOBC, que preconiza a exposição dos insetos a resíduos secos dos agrotóxicos, a fim de facilitar a comparação e discussão de dados obtidos por diferentes autores (DEGRANDE et al., 2002).

Todos os sete fungicidas testados foram inócuos a adultos de C. externa, apresentando uma mortalidade às 120 horas após início da exposição que variou de 0,0 a 16,2\% (Tabela 3). Moura (2007), ao testar o efeito do fungicida/acaricida enxofre em adultos do predador constatou baixa mortalidade, assim como Silva et al. (2006), que classificaram oxicloreto de cobre como inócuo à fase adulta de $C$. externa. A falta de efeito destes compostos deve-se provavelmente ao fato de que os fungicidas testados não apresentam capacidade de atuar em nenhum sítio de ação da fisiologia do predador C. externa.

Os herbicidas glifosato e dicloreto de paraquate proporcionaram mortalidade total de 10,8 e $0,0 \%$, respectivamente (Tabela 3 ), sendo enquadrados na classe 1. Não foram encontrados na literatura estudos sobre efeito de herbicidas sobre adultos de C. externa.

Os testes iniciais de laboratório submetem o inseto a máxima exposição aos resíduos dos agrotóxicos, constituindo-se na condição mais adversa ao inimigo natural. Devido a isto, os agrotóxicos que foram enquadrados como inócuos nestes bioensaios não necessitam mais serem testados, de acordo com a sequência de testes proposta pela IOBC. Entretanto, para obtenção de informações mais completas sobre o efeito destes agrotóxicos em adultos do predador, é recomendada a realização de testes para verificação de possíveis efeitos subletais. Para os inseticidas malationa, dimetoato, fosmete e deltametrina, que apresentaram grau de toxicidade a adultos de C. externa, são recomendados testes de persistência biológica em casa de vegetação e de campo, em pomares de pessegueiro, a fim de prover informações definitivas sobre o efeito ao longo do tempo destes produtos e ajudar a estimar o risco de intoxicação deste inimigo natural. 
TABELA 1 - Caracterização dos agrotóxicos registrados na cultura do pessegueiro e utilizados nos testes de toxicidade sobre a fase adulta de Chrysoperla externa (AGROFIT, 2008).

\begin{tabular}{|c|c|c|c|c|c|c|}
\hline Produto comercial & Ingrediente ativo & Grupo químico & $\mathbf{C}^{1}$ & D.C. ${ }^{2}$ & C.i.a. ${ }^{3}$ & C.f.c. ${ }^{4}$ \\
\hline Agritoato 400 & Dimetoato & Organofosforado & I & 250 & 0,160 & 0,400 \\
\hline Assist* & Óleo mineral 1 & Hidrocarbonetos & I & 2000 & 2,420 & 3,200 \\
\hline Decis $25 \mathrm{EC}$ & Deltametrina & Piretroide & I & 40 & 0,002 & 0,064 \\
\hline Imidan 500 WP* & Fosmete & Organofosforado & I & 200 & 0,160 & 0,320 \\
\hline Malathion 500 EC* & Malationa & Organofosforado & I & 300 & 0,240 & 0,480 \\
\hline Oppa* & Óleo mineral 2 & Hidrocarbonetos & I & 1500 & 1,920 & 2,400 \\
\hline Vertimec $18 \mathrm{EC}^{*}$ & Abamectina & Avermectina & $\mathrm{I} / \mathrm{A}$ & 80 & 0,002 & 0,128 \\
\hline Amistar $500 \mathrm{WG}^{*}$ & Azoxystrobina & Estrobilurina & $\mathrm{F}$ & 20 & 0,016 & 0,032 \\
\hline Cuprozeb* & $\begin{array}{l}\text { Mancozebe }+ \\
\text { Oxicloreto de cobre }\end{array}$ & $\begin{array}{l}\text { Ditiocarbamato + } \\
\text { Inorgânico }\end{array}$ & $\mathrm{F}$ & 200 & $\begin{array}{c}0,140+ \\
0,096\end{array}$ & 0,320 \\
\hline Dodex 450 SC* & Dodina & Guanidina & $\mathrm{F}$ & 175 & 0,126 & 0,280 \\
\hline Folicur $200 \mathrm{EC}^{*}$ & Tebuconazole & Triazol & $\mathrm{F}$ & 100 & 0,320 & 1,600 \\
\hline Folpan Agricur 500 WP* & Folpete & Dicarboximida & $\mathrm{F}$ & 250 & 0,200 & 0,400 \\
\hline Manzate $800^{*}$ & Mancozebe & Ditiocarbamato & $\mathrm{F}$ & 200 & 0,256 & 0,320 \\
\hline Orthocide $500 *$ & Captana & Dicarboximida & $\mathrm{F}$ & 240 & 0,192 & 0,384 \\
\hline Gramoxone & $\begin{array}{l}\text { Dicloreto de } \\
\text { paraquate }\end{array}$ & Bipiridílio & $\mathrm{H}$ & $3^{\ominus}$ & 0,300 & 1,500 \\
\hline Roundup* & Glifosato & Glicina substituída & $\mathrm{H}$ & $6^{\ominus}$ & 1,440 & 3,000 \\
\hline Sumithion 500 EC (Padrão) & Fenitrotiona & Organofosforado & I & 150 & 0,120 & 0,240 \\
\hline
\end{tabular}

*Recomendados na Produção Integrada de Pêssego; ${ }^{1}$ Classe: $\mathrm{I}=$ inseticida, $\mathrm{F}=$ fungicida, $\mathrm{H}=$ herbicida, $\mathrm{A}=\mathrm{acaricida} ;{ }^{2} \mathrm{D} . \mathrm{C} .=\mathrm{Do}-$ sagem da formulação comercial $\left(\mathrm{g}\right.$ ou mL. $\left.100 \mathrm{~L}^{-1}\right){ }^{\ominus} \mathrm{L} . \mathrm{ha}^{-1} ;{ }^{3} \mathrm{C}$. i.a. $=$ Concentração $(\%)$ testada do ingrediente ativo na calda; ${ }^{4} \mathrm{C}$. f.c. $=$ Concentração (\%) testada da formulação comercial na calda.

TABELA 2 - Mortalidade (no \pm EP) acumulada de fêmeas e machos às 24; 72 e 120 horas após início da exposição da fase adulta de Chrysoperla externa a resíduos dos agrotóxicos registrados na cultura do pessegueiro.

\begin{tabular}{|c|c|c|c|c|c|c|c|}
\hline \multirow{2}{*}{ Tratamento } & \multirow{2}{*}{ D.C. ${ }^{*}$} & \multicolumn{2}{|c|}{$M^{1}[24$ horas] } & \multicolumn{2}{|c|}{$\mathrm{M}^{1}[72$ horas $]$} & \multicolumn{2}{|c|}{$\mathrm{M}^{1}[120$ horas $]$} \\
\hline & & q & $\hat{0}$ & 인 & $\hat{0}$ & 인 & $\hat{0}$ \\
\hline \multicolumn{8}{|c|}{ Bioensio I } \\
\hline Testemunha & - & $0,0 \pm 0,0 \mathrm{bA}$ & $0,0 \pm 0,0 \mathrm{cA}$ & $0,0 \pm 0,0 \mathrm{bA}$ & $0,0 \pm 0,0 \mathrm{bA}$ & $0,0 \pm 0,0 \mathrm{bA}$ & $0,0 \pm 0,0 \mathrm{bA}$ \\
\hline Abamectina & 80 & $0,0 \pm 0,0 \mathrm{bA}$ & $0,0 \pm 0,0 \mathrm{cA}$ & $0,0 \pm 0,0 \mathrm{bA}$ & $0,0 \pm 0,0 \mathrm{bA}$ & $0,0 \pm 0,0 \mathrm{bA}$ & $0,0 \pm 0,0 \mathrm{bA}$ \\
\hline Malationa & 300 & $2,5 \pm 0,6 \mathrm{aA}$ & $3,3 \pm 0,5 \mathrm{bA}$ & $4,8 \pm 0,0 \mathrm{aA}$ & $5,0 \pm 0,0 \mathrm{aA}$ & $5,0 \pm 0,0 \mathrm{aA}$ & $5,0 \pm 0,0 \mathrm{aA}$ \\
\hline Fosmete & 200 & $3,3 \pm 1,3 \mathrm{aB}$ & $4,8 \pm 0,5 \mathrm{aA}$ & $5,0 \pm 0,0 \mathrm{aA}$ & $5,0 \pm 0,0 \mathrm{aA}$ & $5,0 \pm 0,0 \mathrm{aA}$ & $5,0 \pm 0,0 \mathrm{aA}$ \\
\hline & 50 & $3,3 \pm 1,0 \mathrm{aB}$ & $4,8 \pm 0,5 \mathrm{aA}$ & $5,0 \pm 0,0 \mathrm{aA}$ & $5,0 \pm 0,0 \mathrm{aA}$ & $5,0 \pm 0,0 \mathrm{aA}$ & $5,0 \pm 0,0 \mathrm{aA}$ \\
\hline & 150 & $2,3 \pm 1,7 \mathrm{aB}$ & $4,5 \pm 0,6 \mathrm{aA}$ & $4,5 \pm 0,6 \mathrm{aA}$ & $5,0 \pm 0,0 \mathrm{aA}$ & $5,0 \pm 0,0 \mathrm{aA}$ & $5,0 \pm 0,0 \mathrm{aA}$ \\
\hline \multicolumn{8}{|c|}{ Bioensio II } \\
\hline Testem & - & $0,0 \pm 0,0 \mathrm{bA}$ & $0,0 \pm 0,0 \mathrm{bA}$ & $0,0 \pm 0,0 \mathrm{bA}$ & $0,0 \pm 0,0 \mathrm{bA}$ & $0,0 \pm 0,0 \mathrm{bA}$ & $0,0 \pm 0,0 \mathrm{cA}$ \\
\hline Óleo mineral 1 & 2000 & $3 \pm 0,5 \mathrm{bA}$ & $0,0 \pm 0,0 \mathrm{bA}$ & $0,3 \pm 0,5 \mathrm{bA}$ & $0,0 \pm 0,0 \mathrm{bA}$ & $0,3 \pm 0,5 \mathrm{bA}$ & $0,3 \pm 0,5 \mathrm{cA}$ \\
\hline Óleo mineral 2 & 1500 & $0 \pm 0,0 \mathrm{bA}$ & $0,0 \pm 0,0 \mathrm{bA}$ & $0,0 \pm 0,0 \mathrm{bA}$ & $0,0 \pm 0,0 \mathrm{bA}$ & $0,0 \pm 0,0 \mathrm{bA}$ & $0,0 \pm 0,0 \mathrm{cA}$ \\
\hline Deltametrina & 40 & $0,0 \pm 0,0 \mathrm{bA}$ & $0,3 \pm 0,5 \mathrm{bA}$ & $0,0 \pm 0,0 \mathrm{bA}$ & $0,8 \pm 1,0 \mathrm{bA}$ & $0,5 \pm 0,6 \mathrm{bB}$ & $2,8 \pm 1,0 \mathrm{bA}$ \\
\hline Tebuconazole & 100 & $0,0 \pm 0,0 \mathrm{bA}$ & $0,0 \pm 0,0 \mathrm{bA}$ & $0,0 \pm 0,0 \mathrm{bA}$ & $0,3 \pm 0,5 \mathrm{bA}$ & $0,0 \pm 0,0 \mathrm{bA}$ & $0,3 \pm 0,5 \mathrm{cA}$ \\
\hline Fenitr & 150 & $3,8 \pm 1,0 \mathrm{aB}$ & $5,0 \pm 0,0 \mathrm{aA}$ & $5,0 \pm 0,0 \mathrm{aA}$ & $5,0 \pm 0,0 \mathrm{aA}$ & $5,0 \pm 0,0 \mathrm{aA}$ & $5,0 \pm 0,0 \mathrm{aA}$ \\
\hline \multicolumn{8}{|c|}{ Bioensaio III } \\
\hline & - & & $0,0 \pm 0,0 \mathrm{bA}$ & $0,0 \pm 0,0 \mathrm{bA}$ & $0,0 \pm 0,0 \mathrm{bA}$ & $0,0 \pm 0,0 \mathrm{bA}$ & $0,3 \pm 0,5 \mathrm{bA}$ \\
\hline strobina & 20 & $0,0 \pm 0,0 \mathrm{bA}$ & $0,0 \pm 0,0 \mathrm{bA}$ & $0,0 \pm 0,0 \mathrm{bA}$ & $0,3 \pm 0,5 \mathrm{bA}$ & $0,0 \pm 0,0 \mathrm{bA}$ & $0,3 \pm 0,5 \mathrm{bA}$ \\
\hline Captana & 240 & $0,3 \pm 0,5 \mathrm{bA}$ & $0,0 \pm 0,0 \mathrm{bA}$ & $0,3 \pm 0,5 \mathrm{bA}$ & $0,0 \pm 0,0 \mathrm{bA}$ & $0,3 \pm 0,5 \mathrm{bA}$ & $0,0 \pm 0,0 \mathrm{bA}$ \\
\hline Dodina & 175 & $0,0 \pm 0,0 \mathrm{bA}$ & $0,0 \pm 0,0 \mathrm{bA}$ & $0,0 \pm 0,0 \mathrm{bA}$ & $0,3 \pm 0,5 \mathrm{bA}$ & $0,3 \pm 0,5 \mathrm{bA}$ & $0,3 \pm 0,5 \mathrm{bA}$ \\
\hline Mancozebe & 200 & $0,0 \pm 0,0 \mathrm{bA}$ & $0,0 \pm 0,0 \mathrm{bA}$ & $0,0 \pm 0,0 \mathrm{bA}$ & $0,0 \pm 0,0 \mathrm{bA}$ & $0,0 \pm 0,0 \mathrm{bA}$ & $0,0 \pm 0,0 \mathrm{bA}$ \\
\hline & 150 & $5,0 \pm 0,0 \mathrm{aA}$ & $5,0 \pm 0,0 \mathrm{aA}$ & $5,0 \pm 0,0 \mathrm{aA}$ & $5,0 \pm 0,0 \mathrm{aA}$ & $5,0 \pm 0,0 \mathrm{aA}$ & $5,0 \pm 0,0$ aA \\
\hline \multicolumn{8}{|c|}{ Bioensaio IV } \\
\hline & - & $0,0 \pm 0,0 \mathrm{bA}$ & $0,0 \pm 0,0 \mathrm{bA}$ & $0,0 \pm 0,0 \mathrm{bA}$ & $0,0 \pm 0,0 \mathrm{cA}$ & $0,0 \pm 0,0 \mathrm{bA}$ & $0,8 \pm 1,0 \mathrm{bA}$ \\
\hline Mancozebe + ox. cobre & 200 & $0,0 \pm 0,0 \mathrm{bA}$ & $0,5 \pm 0,6 \mathrm{bA}$ & $0,3 \pm 0,5 \mathrm{bB}$ & $1,8 \pm 1,5 \mathrm{bA}$ & $0,5 \pm 0,6 \mathrm{bA}$ & $1,8 \pm 1,5 \mathrm{bA}$ \\
\hline Folpete & 250 & $0,0 \pm 0,0 \mathrm{bA}$ & $0,0 \pm 0,0 \mathrm{bA}$ & $0,3 \pm 0,5 \mathrm{bA}$ & $0,3 \pm 0,5 \mathrm{bA}$ & $0,5 \pm 0,6 \mathrm{bA}$ & $0,5 \pm 0,6 \mathrm{bA}$ \\
\hline Glifosato & $6^{\Theta}$ & $0,0 \pm 0,0 \mathrm{bA}$ & $0,5 \pm 0,6 \mathrm{bA}$ & $0,3 \pm 0,5 \mathrm{bA}$ & $0,8 \pm 0,5 \mathrm{bA}$ & $0,3 \pm 0,5 \mathrm{bB}$ & $1,5 \pm 0,6 \mathrm{bA}$ \\
\hline Dicloreto de paraquate & $3^{\ominus}$ & $0,0 \pm 0,0 \mathrm{bA}$ & $0,0 \pm 0,0 \mathrm{bA}$ & $0,0 \pm 0,0 \mathrm{bA}$ & $0,0 \pm 0,0 \mathrm{cA}$ & $0,0 \pm 0,0 \mathrm{bA}$ & $0,3 \pm 0,5 \mathrm{bA}$ \\
\hline Fenitrotiona & 150 & $4,8 \pm 0,5 \mathrm{aA}$ & $5,0 \pm 0,0 \mathrm{aA}$ & $5,0 \pm 0,0 \mathrm{aA}$ & $5,0 \pm 0,0 \mathrm{aA}$ & $5,0 \pm 0,0 \mathrm{aA}$ & $5,0 \pm 0,0 \mathrm{aA}$ \\
\hline
\end{tabular}

*D.C. $=$ Dosagem do produto comercial $\left(\mathrm{g}\right.$ ou mL. $100 \mathrm{~L}^{-1} ;{ }^{\ominus} \mathrm{L}_{\mathrm{h}}$ ha $\left.{ }^{-1}\right) ;{ }^{1}$ Valor médio obtido de quatro repetições com cinco casais cada; Médias seguidas pela mesma letra minúscula nas colunas e maiúscula nas linhas para cada período de avaliação não diferem significativamente entre si, pelo teste de Tukey, a 5\% de probabilidade. 
TABELA 3 - Mortalidade acumulada (no \pm EP e \%) e classificação da IOBC/WPRS às 24; 72 e 120 horas após início da exposição de adultos de Chrysoperla externa a resíduos dos agrotóxicos registrados na cultura do pessegueiro.

\begin{tabular}{|c|c|c|c|c|c|c|c|c|c|c|}
\hline \multirow{2}{*}{ Tratamento } & \multirow{2}{*}{ D.C. ${ }^{*}$} & \multicolumn{3}{|c|}{ M [24 horas] } & \multicolumn{3}{|c|}{ M [72 horas] } & \multicolumn{3}{|c|}{ M [120 horas] } \\
\hline & & $n^{0} \pm \mathbf{E P}^{1}$ & $\%^{* * *}$ & $\mathbf{C}^{* * * *}$ & $n^{0} \pm E^{1}$ & $\%^{* * *}$ & $\mathbf{C}^{* * *}$ & $\mathbf{n}^{0} \pm \mathbf{E P}^{1}$ & $\%^{* *}$ & $\mathbf{C}^{* * *}$ \\
\hline \multicolumn{11}{|c|}{ Bioensaio I } \\
\hline Testemunha & - & $0,0 \pm 0,0 \mathrm{~b}$ & 0,0 & - & $0,0 \pm 0,0 \mathrm{~b}$ & 0,0 & - & $0,0 \pm 0,0 \mathrm{~b}$ & 0,0 & - \\
\hline Abamectina & 80 & $0,0 \pm 0,0 \mathrm{~b}$ & 0,0 & 1 & $0,0 \pm 0,0 \mathrm{~b}$ & 0,0 & 1 & $0,0 \pm 0,0 \mathrm{~b}$ & 0,0 & 1 \\
\hline Malationa & 300 & $5,8 \pm 1,0 \mathrm{a}$ & 57,5 & 2 & $9,8 \pm 0,5 \mathrm{a}$ & 97,5 & 3 & $10,0 \pm 0,0 \mathrm{a}$ & 100,0 & 4 \\
\hline Fosmete & 200 & $8,0 \pm 1,4 \mathrm{a}$ & 80,0 & 3 & $10,0 \pm 0,0 \mathrm{a}$ & 100,0 & 4 & $10,0 \pm 0,0 \mathrm{a}$ & 100,0 & 4 \\
\hline Dime & 250 & $8,0 \pm 1,4 \mathrm{a}$ & 80,0 & 3 & $10,0 \pm 0,0 \mathrm{a}$ & 100,0 & 4 & $10,0 \pm 0,0 \mathrm{a}$ & 100,0 & 4 \\
\hline Fenitr & 150 & $6,8 \pm 2,1 \mathrm{a}$ & 67,5 & 2 & $9,5 \pm 0,6 \mathrm{a}$ & 95,0 & 3 & $10,0 \pm 0,0 \mathrm{a}$ & 100,0 & 4 \\
\hline \multicolumn{11}{|c|}{ Bioensaio II } \\
\hline Testemunha & - & $0,0 \pm 0,0 \mathrm{~b}$ & 0,0 & - & $0,0 \pm 0,0 \mathrm{~b}$ & 0,0 & - & $0,0 \pm 0,0 \mathrm{c}$ & 0,0 & - \\
\hline neral 1 & 2000 & $0,3 \pm 0,5 \mathrm{~b}$ & 2,5 & 1 & $0,3 \pm 0$ & 2,5 & 1 & $0,5 \pm 0,6 \mathrm{c}$ & 5,0 & 1 \\
\hline neral 2 & 1500 & $0,0 \pm 0,0 \mathrm{~b}$ & 0,0 & 1 & $0,0 \pm 0,0 \mathrm{~b}$ & 0,0 & 1 & $0,0 \pm 0,0 \mathrm{c}$ & 0,0 & 1 \\
\hline Deltametrina & 40 & $0,3 \pm 0,5 \mathrm{~b}$ & 2,5 & 1 & $0,8 \pm 1,0 \mathrm{~b}$ & 7,5 & 1 & $3,3 \pm 1,0 \mathrm{~b}$ & 32,5 & 2 \\
\hline Tebuco & 100 & $0,0 \pm 0,0 \mathrm{~b}$ & 0,0 & 1 & $0,3 \pm 0,5 b$ & 2,5 & 1 & $0,3 \pm 0,5 \mathrm{c}$ & 2,5 & 1 \\
\hline Fenitrotiona & 150 & $8,8 \pm 1,0 \mathrm{a}$ & 82,5 & 3 & $10,0 \pm 0,0 \mathrm{a}$ & 100,0 & 4 & $10,0 \pm 0,0 \mathrm{a}$ & 100,0 & 4 \\
\hline \multicolumn{11}{|c|}{ Bioensaio III } \\
\hline Teste & - & $0,0 \pm 0,0 \mathrm{~b}$ & 0,0 & - & $0,0 \pm 0,0 \mathrm{~b}$ & 0,0 & - & $0,3 \pm 0,5 \mathrm{~b}$ & 2,5 & - \\
\hline Azoxystrobina & 20 & $0,0 \pm 0,0 \mathrm{~b}$ & 0,0 & 1 & $0,3 \pm 0,5 \mathrm{~b}$ & 2,5 & 1 & $0,3 \pm 0,5 \mathrm{~b}$ & 0,0 & 1 \\
\hline Captana & 240 & $0,3 \pm 0,5 b$ & 2,5 & 1 & $0,3 \pm 0,5 \mathrm{~b}$ & 2,5 & 1 & $0,3 \pm 0,5 \mathrm{~b}$ & 0,0 & 1 \\
\hline Dodina & 175 & $0,0 \pm 0,0 \mathrm{~b}$ & 0,0 & 1 & $0,3 \pm 0,5 \mathrm{~b}$ & 2,5 & 1 & $0,5 \pm 1,0 \mathrm{~b}$ & 2,6 & 1 \\
\hline Mancozebe & 200 & $0,0 \pm 0,0 \mathrm{~b}$ & 0,0 & 1 & $0,0 \pm 0,0 \mathrm{~b}$ & 0,0 & 1 & $0,0 \pm 0,0 \mathrm{~b}$ & 0,0 & 1 \\
\hline$\underline{\text { Fenitrotiona }}$ & 150 & $10,0 \pm 0,0 \mathrm{a}$ & 100,0 & 4 & $10,0 \pm 0,0 \mathrm{a}$ & 100,0 & 4 & $10,0 \pm 0,0 \mathrm{a}$ & 100,0 & 4 \\
\hline \multicolumn{11}{|c|}{ Bioensaio IV } \\
\hline Testemunha & - & $0,0 \pm 0,0 \mathrm{~b}$ & 0,0 & - & $0,0 \pm 0,0 \mathrm{c}$ & 0,0 & - & $0,8 \pm 1,0 \mathrm{~b}$ & 7,5 & - \\
\hline Mancozebe + ox. cobre & 200 & $0,5 \pm 0,6 \mathrm{~b}$ & 5,0 & 1 & $2,0 \pm 1,4 \mathrm{~b}$ & 20,0 & 1 & $2,3 \pm 1,9 b$ & 16,2 & 1 \\
\hline Folpete & 250 & $0,0 \pm 0,0 \mathrm{~b}$ & 0,0 & 1 & $0,5 \pm 0,6 b c$ & 5,0 & 1 & $1,0 \pm 0,8 b$ & 2,7 & 1 \\
\hline Glifosato & $6^{\ominus}$ & $0,5 \pm 0,6 \mathrm{~b}$ & 5,0 & 1 & $1,0 \pm 0,8 \mathrm{bc}$ & 10,0 & 1 & $1,8 \pm 1,0 \mathrm{~b}$ & 10,8 & 1 \\
\hline Dicloreto de $p$ & $3^{\ominus}$ & $0,0 \pm 0,0 \mathrm{~b}$ & 0,0 & 1 & $0,0 \pm 0,0 \mathrm{c}$ & 0,0 & 1 & $0,3 \pm 0,5 \mathrm{~b}$ & 0,0 & 1 \\
\hline Fenitrotiona & 150 & $9,8 \pm 0,5 \mathrm{a}$ & 97,5 & 3 & $10,0 \pm 0,0 \mathrm{a}$ & 100,0 & 4 & $10,0 \pm 0,0 \mathrm{a}$ & 100,0 & 4 \\
\hline
\end{tabular}

*D.C. $=$ Dosagem do produto comercial $\left(\mathrm{g}\right.$ ou mL $\left..100 \mathrm{~L}^{-1} ;{ }^{\ominus} \mathrm{L} \cdot \mathrm{ha}^{-1}\right) ; * *$ Mortalidade corrigida por Schneider- Orelli; ***C $=\mathrm{Classes}$ da IOBC/WPRS, $1=$ inócuo $(<30 \%) ; 2=$ levemente nocivo (30-79\%); $3=$ moderadamente nocivo (80-99\%); $4=$ nocivo ( $>99 \%)$; ${ }^{1}$ Valor médio obtido de quatro repetições com cinco casais cada. Médias seguidas pela mesma letra nas colunas, para cada período de avaliação, não diferem significativamente entre si, pelo teste de Tukey, a $5 \%$ de probabilidade.

\section{CONCLUSÃO}

Para os agrotóxicos, nas concentrações (\% de ingrediente ativo) avaliadas, conclui-se que: os fungicidas azoxystrobina $(0,016)$, captana $(0,192)$, dodina $(0,126)$, folpete $(0,200)$, mancozebe $(0,256)$, mancozebe + oxicloreto de cobre $(0,140+0,096) \mathrm{e}$ tebuconazole $(0,320)$, o inseticida/acaricida abamectina $(0,002)$, os inseticidas óleo mineral $1(2,420)$ e óleo mineral $2(1,920)$, e os herbicidas dicloreto de paraquate $(0,300)$ e glifosato $(1,440)$ são inócuos aos adultos do predador. $\mathrm{O}$ inseticida deltametrina $(0,002)$ é levemente nocivo, e os inseticidas dimetoato $(0,160)$, fosmete $(0,160)$ e malationa $(0,240)$ são nocivos à fase adulta de $C$. externa em testes de exposição residual em laboratório.

\section{REFERÊNCIAS}

AGROFIT. Sistema de agrotóxicos fitossanitários. Disponivel em: http://agrofit.agricultura.gov.br/ agrofit_cons/principal_agrofit_cons. Acesso em: maio 2008 .

BOTTON, M.; ARIOLI, C.J.; MASCARO, F.A. Manejo de pragas na cultura do pessegueiro. In: ENCONTRO NACIONAL DE FRUTICULTURA E CLIMA TEMPERADO, 8., 2005. Fraiburgo. Anais... Fraiburgo: EPAGRI, 2005. v.1, p 155-159. 
CARVALHO, C.F.; SOUZA, B. Métodos de criação e produção de crisopídeos. In: BUENO, V.H.P. (Ed.). Controle biológico de pragas: produção massal e controle de qualidade. Lavras: UFLA, 2000. p. 91-109.

CARVALHO, G.A. Seletividade de produtos fitossanitários a parasitoides e predadores. In: SEMINÁRIO BRASILEIRO DE PRODUÇÃO INTEGRADA DE FRUTAS, 4., 2002, Bento Gonçalves. Anais...Bento Gonçalves: Embrapa Uva e Vinho, 2002. p.49-51.

CASTRO, A.L.G.; CRUZ, I.; SILVA, I.F.; FERREIRA, T.E.; LEÃO, M.L.; PAULA, C.S. Ciclo biológico de Chrysoperla externa (Hagen, 1861) (Neuroptera: Chrysopidae) em condições de laboratório. In: CONGRESSO DE ECOLOGIA DO BRASIL, 9., 2009. São Lourenço. Anais... p.1-3.

DE BORTOLI, S.A.; CAETANO, A.C.; MURATA, A.T.; OLIVEIRA, J.E.M. Desenvolvimento e capacidade predatória de Chrysoperla externa (Hagen) (Neuroptera: Chrysopidae) em diferentes presas. Revista de Biologia e Ciências da Terra, Paraíba, v. 6, n. 1, p. 145-152, 2006.

DEGRANDE, P.E.; REIS, P.R.; CARVALHO, G.A.; BELARMINO, L.C. Metodologia para avaliar o impacto de pesticidas sobre inimigos naturais. In: PARRA, J.R.P.; BOTELHO, P.S.M.; CORRÊAFERREIRA, B.S.; BENTO, J.M.S. (Ed.). Controle biológico no Brasil: parasitoides e predadores. São Paulo: Manole, 2002. cap.5, p.71-93.

GIOLO, F.P.; MEDINA, P.; GRÜTZMACHER, A.D.; VIÑUELA, E. Effects of pesticides commonly used in peach orchards in Brazil on predatory lacewing Chrysoperla carnea under laboratory conditions. BioControl, Dordrecht, v.54, p.625-635, 2009.

GODOY, M.S.; CARVALHO, G.A.; MORAES, J.C.; COSME, L.V.; GOUSSAIN, M.M.; CARVALHO, C.F.; MORAIS, A.A. Seletividade de seis inseticidas utilizados em citros a pupas e adultos de Chrysoperla externa (Hagen) (Neuroptera: Chrysopidae). Neotropical Entomology, Londrina, v.33, n.3, p.359-364, 2004.

IBGE. Banco de dados. Disponível em: < http:// www.ibge.gov.br/estadosat/ $>$. Acesso em: 12 jan. 2010 .
MACHADO, A.A.; CONCEIÇÃO, A.R. WinStat: sistema de análise estatística para windows. Universidade Federal de Pelotas, 2007. Disponível em $<$ http://www.ufpel.edu.br/ machado $>$.

MOURA, A.P. Efeitos de produtos fitossanitários utilizados na produção integrada de maçã sobre Chrysoperla externa (Hagen, 1861) (Neuroptera: Chrysopidae). 2007, 109 f. Tese (Doutorado em Entomologia) - Universidade Federal de Lavras, Lavras, 2007.

MOURA, A.P.; CARVALHO, G.A.; MOSCARDINI, V.F.; MARQUES, M.C.; SOUZA, J.R. Toxicidade de pesticidas recomendados na Produção Integrada de Maçã (PIM) a populações de Chrysoperla externa (Hagen) (Neuroptera: Chrysopidae). Neotropical Entomology, Londrina, v.38, n.5, p. 395-404, 2009.

NTEPIP. Normas Técnicas Específicas para a Produção Integrada de Prunus spp. Disponível em: < http://www.ufpel.tche.br/pif/>. Acesso em: 30 out. 2009.

PÜNTENER, W. Manual for field trials in plant protection. $2^{\text {nd }}$ ed. Greensboro: CibaGeigy,Agricultural Division, 1981.

ROCHA, L.C.D. Seletividade fisiológica de inseticidas utilizados em cultura cafeeira sobre os predadores Chrysoperla externa (Hagen, 1861) (Neuroptera: Chrysopidae) e Cryptolaemus montrouzieri Mulsant, 1853 (Coleoptera: Coccinellidae). 2008. 133f. Tese (Doutorado em Entomologia) - Universidade Federal de Lavras, Lavras, 2008.

SCHUBER, J.M.; MONTEIRO, L.B.; POLTRONIERI, A.S.; CARDOSO, N.A.; MIO, L.L. Influência de sistemas de produção sobre a ocorrência de inimigos naturais de afídeos em pomares de pessegueiros em Araucária - PR. Revista Brasileira de Fruticultura, Jaboticabal, v.30, n.2, p.336-342, 2008.

SILVA, R.A.; CARVALHO, G.A.; CARVALHO, C.F.; REIS, P.R.; SOUZA, B.; PEREIRA, A.M. Ação de produtos fitossanitários utilizados em cafeeiros sobre pupas e adultos de Chrysoperla externa (Hagen, 1861) (Neuroptera: Chrysopidae). Ciência Rural, Santa Maria, v.36, n.1, p.8-14, 2006. 
VILELA, M. Seletividade de acaricidas usados em cafeeiro para Chrysoperla externa (Hagen, 1861) (Neuroptera: Chrysopidae). 2009. 67 f. Dissertação (Mestrado em Entomologia) - Universidade Federal de Lavras, Lavras, 2009.

VOGT, H. Effects of Quassia products on Chrysoperla carnea (Stephens) (Neuroptera, Chrysopidae). IOBC/WPRS Bulletin, Dossenheim, v. 24, n. 4, p. 47-52, 2001.
VOGT, H.; BIGLER, F.; BROWN, K.; CANDOLFI, M.P.; KEMMETER, F.; KÜHNER, C.; MOLL, M.; TRAVIS, A.; UFER, A.; VIÑUELA, E.; WLADBURGER, M.; WALTERSDORFER, A. Laboratory method to test effects of plant protection products on larvae of Chrysoperla carnea (Neuroptera: Chrysopidae). In: CANDOLFI, M.P.; BLUMEL, S.; FORSTER, R.; BAKKER, F.M.; GRIMM, C.; HASSAN S.A.; HEIMBACH, U.; MEAD-BRIGGS, M.A.; REBER, B.; SCHMUCK, R.; VOGT, H. (Ed.). Guidelines to evaluate side-effects of plant protection products to non-target arthropods. Reinheim: IOBC/ WPRS, 2000. p.27-44. 